\title{
Prediction of multiple-feature effects in plasma etching
}

\author{
Gyeong S. Hwang and Konstantinos P. Giapis ${ }^{\text {a) }}$ \\ Division of Chemistry and Chemical Engineering, California Institute of Technology, Pasadena, \\ California 91125
}

(Received 13 January 1997; accepted for publication 3 March 1997)

\begin{abstract}
Charging and topography evolution simulations during plasma etching of dense line-and-space patterns reveal that multiple-feature effects influence critically the etch profile characteristics of the various lines. By including neighboring lines, the simulation predicts a peculiar notching behavior, where the extent of notching varies with the location of the line. Feature-scale modeling can no longer be focused on individual features alone; "adjacency" effects are crucial for understanding and predicting the outcome of etching experiments at reduced device dimensions. (C) 1997 American Institute of Physics. [S0003-6951(97)00318-5]
\end{abstract}

As device dimensions continue to shrink, recipe development for modern etchers is becoming increasingly limited by the cost and time required to find processing windows for desirable etch profiles. Accurate feature-scale simulations are urgently needed to understand the limitations of existing etch tools and to design new generations of reactors. In order to be predictive, such simulations must go beyond the individual feature to capture "adjacency" and wafer-scale effects. ${ }^{1}$ For example, in modeling trench profile evolution, neighboring trenches are typically ignored. When charging effects are important, such simplifications are no longer acceptable. Indeed, the "notching" effect ${ }^{2,3}$ has beautifully demonstrated that profile evolution at the outermost trench can be dramatically different from that obtained in neighboring trenches. For significant electron shadowing in dense, isolated line-and-space (L\&S) metal-oxide-semiconductor structures, it has been shown ${ }^{4}$ that a potential difference between the lines bordering the outermost trench causes an asymmetric potential distribution in the trench space, which distorts ion trajectories and leads to notching typically at the inner sidewall of the outermost line. Since notching in neighboring isolated lines has not been reported yet, modeling studies need not focus but on the outermost trench. However, when electron shadowing is reduced - as is the case for thin hard masks-our simulations predict notch formation in neighboring lines even when not electrically connected to the edge line or the substrate. Furthermore, the extent of notching depends on the location of the line along the pattern. This peculiar notching behavior is a result of the perturbation of trench charging potentials on local ion and electron dynamics, which alter sidewall potentials that, in turn, influence charging in neighboring trenches.

Typical conditions for modern high-density plasma reactors ${ }^{3}$ are assumed: low pressure $(<10$ mTorr $)$, uniform chlorine plasma of density $1 \times 10^{12} \mathrm{~cm}^{-3}, 100 \%$ dissociation. The wafer electrode is biased; the sheath voltage is given by $37+30 \sin \omega t \mathrm{~V}$, where $\omega=400 \mathrm{kHz}$ is the rf bias frequency. The ion and electron temperatures are assumed to be 0.5 and $4.0 \mathrm{eV}$, respectively. The simulation starts with a perfectly etched structure, consisting of four isolated 0.3 $\mu \mathrm{m}$ lines separated by $0.3 \mu \mathrm{m}$ spaces (trenches) and borders widely open areas (Fig. 1). Each line consists of $0.1 \mu \mathrm{m}$

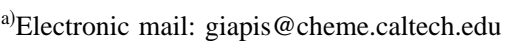

$\mathrm{SiO}_{2}$ onto $0.4 \mu \mathrm{m}$ doped polycrystalline $\mathrm{Si}$ (poly $\mathrm{Si}$ ). The features are formed on top of a uniform layer of $\mathrm{SiO}_{2}$, thick enough $(>100 \mathrm{~nm})$ to prevent tunneling currents to the $\mathrm{Si}$ substrate. Albeit not representative of $0.3 \mu \mathrm{m}$ device rule, this structure is suitable for the study of multiple-feature effects that could be tested experimentally. In addition, if aspect ratio scaling holds, the latter structure could be representative of the $0.15 \mu \mathrm{m}$ device rule, by forming a $50 \mathrm{~nm}$ hard mask $\left(\mathrm{SiO}_{2}\right)$ onto $0.2 \mu \mathrm{m}$ poly $\mathrm{Si}$. The "overetch" step is simulated, referring to prolonged etching after the poly-Si on unpatterned areas is cleared.

Understanding charging damage requires detailed modeling and simulation of ion and electron dynamics in plasmas, through sheaths, and, as they impinge at various microstructure surfaces, where they cause charging, physical sputtering and chemical reactions, or undergo inelastic scattering. A predictive feature-scale simulation that discusses these steps and simplified models to describe them has been presented elsewhere. ${ }^{4}$ Briefly, charging is decoupled from etching for the short time required for the former to reach steady state. Realistic ion and electron energy and angular distributions at the wafer are calculated from sheath theory, based on nonlinear electric fields. Then, charged particles are followed as they impinge on various surfaces, where they transfer their charge. Charge deposition creates local electric fields which, in turn, alter ion trajectories. The Laplace equation is solved iteratively in the microstructure spaces to account for the evolution of the electric fields as more charge accumulates. Steady state is established when the potential distribution along the bottom $\mathrm{SiO}_{2}$ surfaces no longer
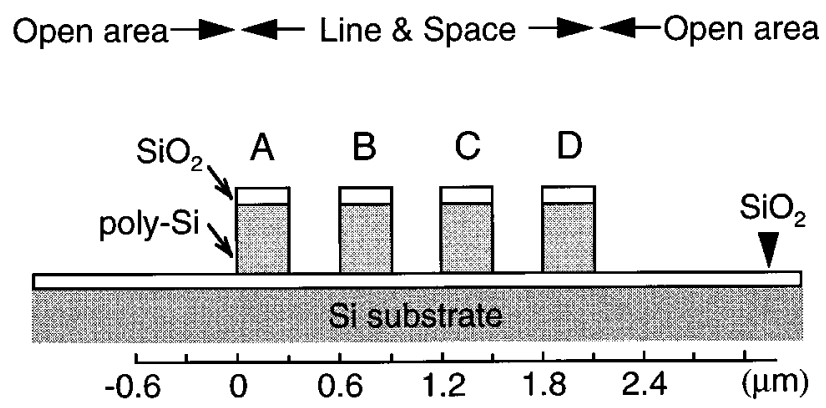

FIG. 1. Schematic depiction of the line-and-space structure considered in the simulation. The open areas are assumed to be infinite. 

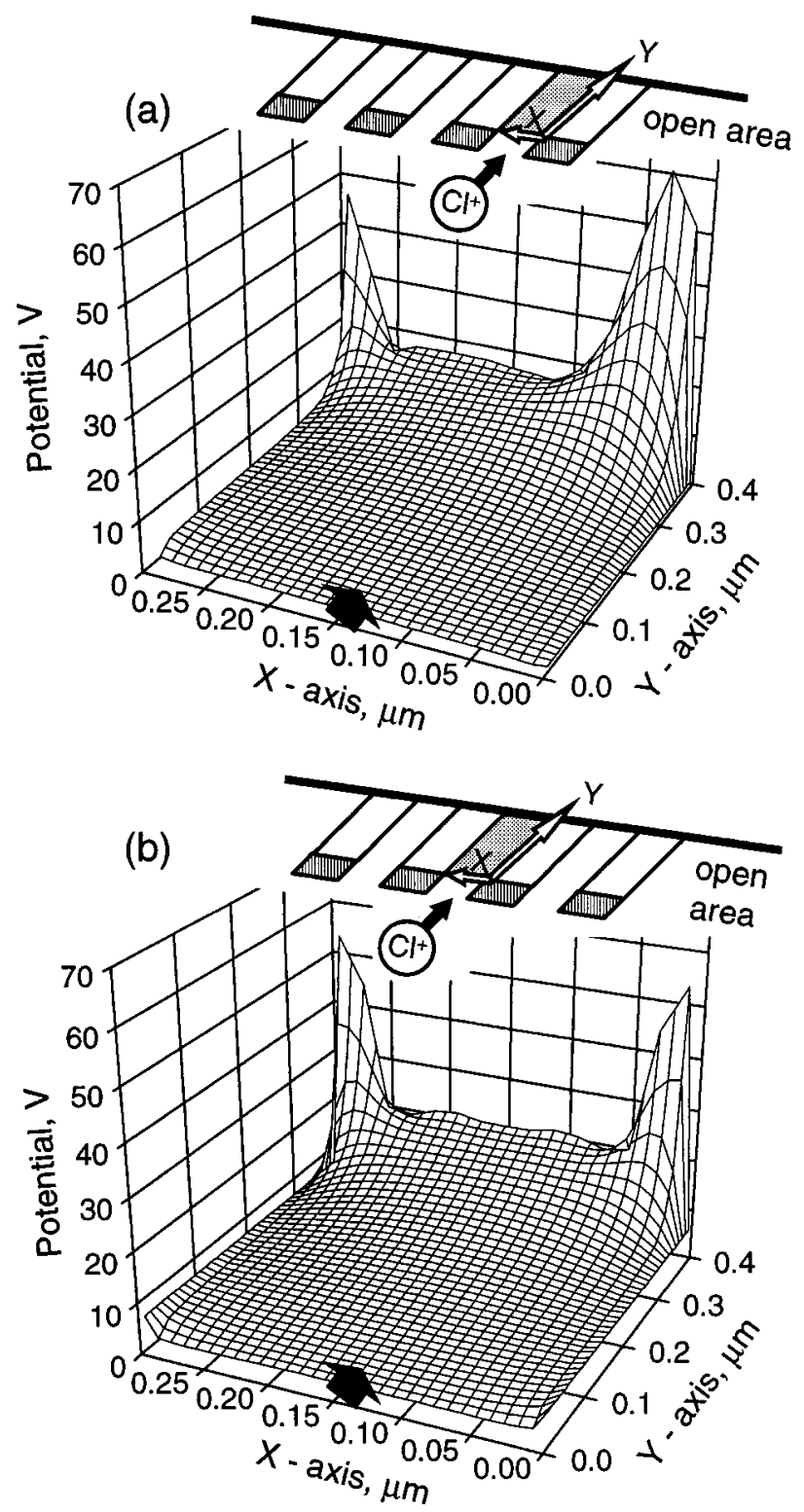

FIG. 2. Three-dimensional charging potential distributions in the trench area bordered by the poly-Si sidewalls for (a) the edge trench, and (b) the intermediate trench. The inset illustrates the area of interest and defines the origin for the potential surface. The microstructure has been rotated to allow for a more convenient description of ion motion in the trench. The arrows show the direction of ions as they approach the potential surface.

change. Surface currents and secondary electron emission are both neglected.

The steady-state charging potential distribution in the various trenches reveals the perturbation in the local ion dynamics occurring as a result of surface charging (Fig. 2). Gradients on this potential surface are a measure of the electric field that influences ion motion. As previously shown, ${ }^{4}$ an asymmetric potential distribution, with a pronounced peak near the inner sidewall foot of the edge line, is critical for notch formation. Remarkably, the potential distribution in the trench between features $C$ and $D$ (the "edge" trench) has two maxima, each located near a poly-Si sidewall foot [Fig. 2(a)]. Note that the two peaks are not symmetric. The peak by the outermost line is larger and broader. These observations forecast the formation of a larger notch at the inner side of the edge line ( $D$-left) than at the neighboring line $(C$ right). But how is it possible to have two potential distribution maxima? Key to the formation of a potential maximum at the trench bottom is a low poly Si potential. When electron shadowing is significant - as is the case for thick photoresist masks - only the outermost lines can maintain a low potential because they receive electrons from the side facing the open area. ${ }^{5}$ All other lines in an isolated L\&S structure acquire a much larger potential since few electrons can be supplied through the trench entrance. A potential maximum forms near the inner sidewall foot of the outermost line as a result of the deflection of ion trajectories towards the lower potential of that line. When thinner masks are used, electron shadowing is reduced; therefore, more electrons can be supplied to the intermediate poly-Si lines through the trench openings, decreasing their potentials. The two peaks of Fig. 2(a) are not identical as a result of the inequality between the potential of the edge line $(1.24 \mathrm{~V})$ and that of the neighboring one $(6.64 \mathrm{~V})$.

The potential distribution in the trench between features $B$ and $C$ (the "middle" trench) is also bimodal [Fig. 2(b)]. This surprising result can likewise be explained by the relatively low equipotentials of the bordering poly-Si lines. The ions entering the middle trench feel the low sidewall potentials and get deflected in the same manner since there is no difference between these two potentials. As more ions impinge near the feet of the sidewalls, the charging potential distribution develops two identical peaks there. Thus, symmetric notches should appear at the sidewall feet of the middle trench. Note also that these peaks are intermediate in magnitude between the two peaks of the potential distribution in the edge trench. This observation has important implications for the notch depth in the middle trench.

The steady-state charging potentials determine the ion dynamics in the microstructure. Reactions at the poly-Si sidewalls and scattering at the $\mathrm{SiO}_{2}$ surface determine how the profile will evolve. The profile evolution simulation combines the ion dynamics with reactions and scattering and is performed as described elsewhere. ${ }^{4}$ As etching of the sidewall proceeds, charging of the exposed $\mathrm{SiO}_{2}$ leads to significant forward deflection of energetic ions which contribute to notching. Thus, the etching simulation becomes coupled to transient charging in the etched area, further complicating the calculation. The problem becomes tractable by solving for a new potential distribution each and every time the notch apex is advanced to the next cell layer. Sputtering and chemical etching of $\mathrm{SiO}_{2}$ are presumed to be insignificant. Etching of the poly-Si is assumed to be ion-limited; spontaneous etching by $\mathrm{Cl}$ atoms is neglected. Material is removed from the surface by collision-induced desorption of chlorinated $\mathrm{SiCl}_{x}$ moieties; the energy and angular dependence of the etch yield has been discussed before. ${ }^{4}$ Inelastic scattering on poly-Si is neglected as most of the scattered atoms impinge subsequently on $\mathrm{SiO}_{2}$. However, direct inelastic scattering on the $\mathrm{Cl}$-covered $\mathrm{SiO}_{2}$ surface must be included as all of the scattered atoms impinge subsequently on poly-Si. Only specular reflection is considered with an energy transfer that follows hard-sphere collision kinematics ${ }^{6}$ with a gasatom to surface mass ratio of 1.0.

Profile evolution simulations have been performed and 


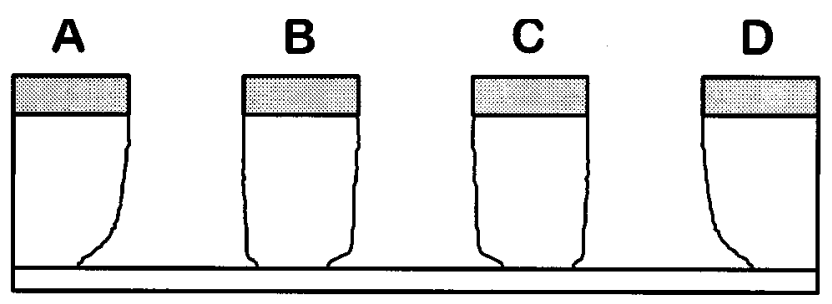

FIG. 3. Notch profiles at the sidewalls of the various lines, predicted for $45 \%$ notching of the edge line. Etching of the outer sidewalls of the edge lines is not simulated. The aspect ratio has been preserved.

the profiles obtained for $45 \%$ notching $^{7}$ at the edge line are shown in Fig. 3. Remarkably, notching is observed at all lines! As anticipated, the notch by the edge lines (A-right and $D$-left) is the widest and deepest; the notches in the middle trench ( $B$-right and $C$-left) are symmetric and less deep than the one of the edge line. The notch at the sidewall facing the edge line ( $B$-left and $C$-right) is the smallest of them all. These observations are fully justifiable in view of the potential distribution in the corresponding trenches (see Fig. 2). Calculation of the normalized flux ${ }^{8}$ and average energy for ions impinging at the various sidewalls, listed in Table I, provides further insight into how the charging potentials can lead to this peculiar notching behavior. The larger potential maximum by the edge line ( $D$-left) facilitates deflection of more ions towards the sidewall with significantly higher translational energy than at any other sidewall. The magnitude and width of the potential maximum at the other lines determines likewise the flux and average energy of ions impinging at the corresponding sidewall. Also note that the broader and larger the potential maximum, the wider the notch. This observation can be explained by the deflection of ions further away from the trench bottom than in cases of smaller potential maxima, where narrower and sharper notches are expected. ${ }^{5}$

In conclusion, charging and etching simulations in low aspect ratio $L \& S$ structures with minimal electron shadowing predict that notching will affect all lines even when electrically isolated. The notch depth and width is found to depend on the location of the sidewall along the L\&S structure. The notch by the inner side of the edge line is the deepest; how-
TABLE I. The normalized flux and average energy of ions bombarding the indicated poly-Si sidewalls.

\begin{tabular}{lccc}
\hline \hline \multicolumn{1}{c}{ Sidewall } & $C$-left & $C$-right & $D$-left \\
\hline Ion flux (a.u.) & 0.202 & 0.159 & 0.326 \\
Avg. energy (eV) & 17.75 & 15.22 & 31.57 \\
\hline \hline
\end{tabular}

ever, the notch at sidewalls of intermediate lines is predicted to be deeper than the notch of the sidewall facing the edge line. These results apply when no tunneling current flows through the gate oxide and, thus, present a worst-case scenario. Nevertheless, they clearly demonstrate that it is no longer sufficient for feature-scale simulations to be focused on individual features. Multiple-feature effects may exist that could explain seemingly peculiar behavior of profile phenomena during plasma etching, in particular when charging effects are important.

Note added in proof: Notches at isolated intermediate lines of features with thinner masks (low aspect ratio) larger electron irradiance of the poly-Si sidewalls-have been reported by A. Hasegawa, Y. Hikosaka, K. Hashimoto, and M. Nakamura, Proceedings of Symposium on Dry Process, Tokyo, Japan, November 1996 (unpublished), pp. 4348. Remarkably, the trend in notch depths seen at the various lines was as predicted in our simulation.

This material was based upon work supported by an NSF Career Award to KFG (CTS-9623450).

${ }^{1}$ D. B. Graves, M. J. Kushner, J. W. Gallagher, A. Garscadden, G. S. Oehrlein, and A. V. Phelps, Database Needs for Modeling and Simulation of Plasma Processing (National Academy, Washington, DC, 1996).

${ }^{2}$ N. Fujiwara, T. Maruyama, and M. Yoneda, Jpn. J. Appl. Phys. 34, 2095 (1995); 35, 2450 (1996).

${ }^{3}$ T. Nozawa, T. Kinoshita, T. Nishizuka, A. Narai, T. Inoue, and A. Nakaue, Jpn. J. Appl. Phys. 34, 2107 (1995).

${ }^{4}$ G. S. Hwang and K. P. Giapis, J. Vac. Sci. Technol. B 15, 70 (1997).

${ }^{5}$ G. S. Hwang and K. P. Giapis (unpublished).

${ }^{6}$ G. S. Hwang, C. M. Anderson, M. J. Gordon, T. A. Moore, T. K. Minton, and K. P. Giapis, Phys. Rev. Lett. 77, 3049 (1996).

${ }^{7}$ No overetching time is stated because it is impossible to calibrate the reactive ion flux without an etching experiment of a structure similar to the simulated one.

${ }^{8}$ The flux has been normalized with respect to the total ion flux entering each trench. 